\title{
The $(\sigma+1)$-Edge-Connectivity Augmentation Problem without Creating Multiple Edges of a Graph
}

\author{
Satoshi Taoka and Toshimasa Watanabe \\ Department of Circuits and Systems, Faculty of Engineering, \\ Hiroshima University \\ 1-4-1, Kagamiyama, Higashi-Hiroshima, 739-8527 Japan \\ Phone: +81-824-24-7666 (Taoka), -7662 (Watanabe) \\ Facsimile: +81-824-22-7028 \\ E-mail : \{taoka, watanabe\}@infonets.hiroshima-u.ac.jp
}

\begin{abstract}
The unweighted $k$-edge-connectivity augmentation problem ( $k$ ECA for short) is defined by "Given a $\sigma$-edge-connected graph $G=$ $(V, E)$, find an edge set $E^{\prime}$ of minimum cardinality such that $G^{\prime}=(V, E \cup$ $\left.E^{\prime}\right)$ is $(\sigma+\delta)$-edge-connected and $\sigma+\delta=k "$, where $E^{\prime}$ is called a solution to the problem. Let $k \mathrm{ECA}(\mathrm{S}, \mathrm{SA})$ denote $k \mathrm{ECA}$ such that both $G$ and $G^{\prime}$ are simple.

The subject of the present paper is $(\sigma+1) \mathrm{ECA}(\mathrm{S}, \mathrm{SA})$ (or $k \mathrm{ECA}(\mathrm{S}, \mathrm{SA})$ with $k=\sigma+1)$. Let $\mathcal{M}$ be any maximum matching of a certain graph $R(G)$ whose vertex set $V_{R}$ consists of vertices representing all leaves of $G$. From $\mathcal{M}$ we obtain an edge set $E_{0}^{\prime}$, with $\left|E_{0}^{\prime}\right|=|\mathcal{M}|$, such that each edge connects vertices in distinct leaves of $G$. Let $\mathcal{L}_{1}$ be the set of leaves to be created by adding $E_{0}^{\prime}$ to $G$, and $\mathcal{K}_{1}$ the set of remaining leaves of $G$.

The main result is to propose two $O\left(\sigma^{2}|V| \log (|V| / \sigma)+|E|+\left|V_{R}\right|^{2}\right)$ time algorithms for finding the following solutions: (1) an optimum solution if $G$ has at least $2 \sigma+6$ leaves or if $\left|\mathcal{L}_{1}\right| \leq\left|\mathcal{K}_{1}\right|$ and $G$ has less than $2 \sigma+6$ leaves; (2) a $\frac{3}{2}$-approximate solution if $\left|\mathcal{L}_{1}\right|>\left|\mathcal{K}_{1}\right|$ and $G$ has less than $2 \sigma+6$ leaves.
\end{abstract}

\section{Introduction}

The unweighted $k$-edge-connectivity augmentation problem ( $k \mathrm{ECA}$ for short) is described as follows: "Given a $\sigma$-edge-connected graph $G=(V, E)$, find an edge set $E^{\prime}$ of minimum cardinality such that $G^{\prime}=\left(V, E \cup E^{\prime}\right)$ is $(\sigma+\delta)$-edgeconnected and $\sigma+\delta=k$." We often denote $G^{\prime}$ as $G+E^{\prime}$, and $E^{\prime}$ is called a solution to the problem. Let $k \mathrm{ECA}\left(*{ }^{* *}\right)$ denote $k \mathrm{ECA}$ with the following restriction (i) and (ii) on $G$ and $E^{\prime}$, respectively: (i) * is set to $S$ if $G$ is required to be simple, and * is left to mean that $G$ may be a multiple graph; (ii) ** is set to MA if creation of new multiple edges in constructing $G^{\prime}$ is allowed, and is set to SA otherwise. In $k \mathrm{ECA}\left({ }^{*}, \mathrm{SA}\right)$, if $G$ is simple then so is $G^{\prime}$, or if $G$ has multiple edges then any multiple edge of $G^{\prime}$ exists in $G$. As for $k \mathrm{ECA}$, 
$k \mathrm{ECA}\left({ }^{*}, \mathrm{MA}\right)$ has mainly been discussed so far. See $355|781213202122| 23$ for the results. It is natural for us to assume that $|V| \geq \sigma+2$ in $(\sigma+1) \operatorname{ECA}(\mathrm{S}, \mathrm{SA})$ : in $(\sigma+1) \operatorname{ECA}(*, S A)$, we may have $|V| \leq \sigma+1$.

As related results, $k \mathrm{ECA}(\mathrm{S}, \mathrm{SA})$ for $G$ having no edges was first discussed in [6], where the problem that is more general than $k \mathrm{ECA}(\mathrm{S}, \mathrm{SA})$ is considered. An $O(|V|+|E|)$ algorithm for $2 \mathrm{ECA}(\mathrm{S}, \mathrm{SA})$ can be obtained by slightly modifying the one given in [3] for $2 \mathrm{ECA}\left({ }^{*}, \mathrm{MA}\right)$. As for $3 \mathrm{ECA}\left({ }^{*}, \mathrm{SA}\right), 23$ ] proposed an $O(|V|+$ $|E|)$ algorithm for $3 \mathrm{ECA}\left({ }^{*}, \mathrm{MA}\right)$, and showed that if $|V| \geq 4$ then this algorithm finds an optimum solution to $3 \mathrm{ECA}(*, \mathrm{SA})$. Concerning $(\sigma+1) \mathrm{ECA}(\mathrm{S}, \mathrm{SA})$ with $|V| \geq \sigma+2$ for $\sigma \in\{3,4\}$, [15] proposed an $O(|V| \log |V|+|E|)$ algorithm. Other related results have been reported in [1416]. T. Jordán showed in [10] that $k \mathrm{ECA}(\mathrm{S}, \mathrm{SA})$ is NP-hard in general, and 2] proposed an $O\left(|V|^{4}\right)$ algorithm for $k \mathrm{ECA}(\mathrm{S}, \mathrm{SA})$ for any fixed $k$.

The subject of the present paper is $(\sigma+1) \mathrm{ECA}(\mathrm{S}, \mathrm{SA})$, that is, $k \mathrm{ECA}(\mathrm{S}, \mathrm{SA})$ with $k=\sigma+1$. Let $\mathcal{M}$ be any maximum matching of the leaf-graph $R(G)$ whose vertex set $V_{R}$ consists of vertices representing all leaves of $G$. (The definition of $R(G)$ is going to be given later). From $\mathcal{M}$ we obtain a certain edge set $E_{0}^{\prime}$, with $\left|E_{0}^{\prime}\right|=|\mathcal{M}|$, such that each edge connects vertices in distinct leaves of $G$. Let $\mathcal{L}_{1}$ be the set of leaves to be created by adding $E_{0}^{\prime}$ to $G$, and $\mathcal{K}_{1}$ the set of remaining leaves of $G$.

The main result of the paper is to propose two $O\left(\sigma^{2}|V| \log (|V| / \sigma)+|E|+\right.$ $\left.\left|V_{R}\right|^{2}\right)$ time algorithms for finding the following solutions for $(\sigma+1) \operatorname{ECA}(\mathrm{S}, \mathrm{SA})$ :

(1) an optimum solution if $G$ has at least $2 \sigma+6$ leaves or if $\left|\mathcal{L}_{1}\right| \leq\left|\mathcal{K}_{1}\right|$ and $G$ has less than $2 \sigma+6$ leaves;

(2) a $\frac{3}{2}$-approximate solution if $\left|\mathcal{L}_{1}\right|>\left|\mathcal{K}_{1}\right|$ and $G$ has less than $2 \sigma+6$ leaves.

A central concept in solving $k \mathrm{ECA}$ is a $t$-edge-connected component of $G$ : a maximal set of vertices such that $G$ has at least $t$ edge-disjoint paths between any pair of vertices in the set [22]. A $t$-edge-connected component whose degree (the number of edges connecting vertices in the set to those outside of it) is equal to the edge-connectivity of $G$ is called a leaf. Although $(\sigma+1) \mathrm{ECA}(\mathrm{S}, \mathrm{SA})$ can be solved almost similarly to general $k \mathrm{ECA}(*, \mathrm{MA})$, the only difference is that the augmenting step has to choose a pair of leaves, each containing a vertex such that they are not adjacent in $G$. (Such a pair of leaves is called a nonadjacent pair.) This requires addition of some other characteristics or processes in finding solutions by means of structural graphs: a structural graph is introduced in [1], and is used as a useful tool that reduces time complexity in finding a solution to $k \mathrm{ECA}\left({ }^{*}, \mathrm{MA}\right)$ in 7,13 .

This paper adopts the operation, called edge-interchange, in finding a solution, where it was introduced in [2021] in order to reduce time complexity of [22]. A set of two nonadjacent pairs of leaves is called a D-combination if they are disjoint. The augmenting step in solving $(\sigma+1) \mathrm{ECA}(\mathrm{S}, \mathrm{SA})$ repeats both choosing a nonadjacent pair of leaves and enlarging a $(\sigma+1)$-edge-connected component by means of edge-interchange (or an analogous operation). Hence 
obtaining an optimum solution requires finding a maximum set of nonadjacent pairs of leaves such that any two members in the set form a D-combination and, therefore, this is reduced to finding a maximum matching of the leaf-graph $R(G)$ of $G$. The point of $(\sigma+1) \operatorname{ECA}(\mathrm{S}, \mathrm{SA})$ is that a solution $E^{\prime}$ is closely related to a maximum matching $\mathcal{M}$ of $R(G)$.

The paper is organized as follows. Basic definitions and several basic results on $\sigma$-edge-connected componets and leaf-graphs are given in Section 2 In Section 3, results on maximum matchings of leaf-graphs are briefly mentioned. Edge-interchange operation is explained in Section 4 Section 5 discusses $(\sigma+1) \operatorname{ECA}(\mathrm{S}, \mathrm{SA})$ when $G$ has less than $2 \sigma+6$ leaves, and Section 6 considers $(\sigma+1) \operatorname{ECA}(\mathrm{S}, \mathrm{SA})$ when $G$ has at least $2 \sigma+6$ leaves.

All proofs are omitted becase of space limitation.

\section{Preliminaries}

\subsection{Basic Definitions}

Technical terms not specified here can be identified in [14919. An undirected graph $G=(V(G), E(G))$ consists of a finite and nonempty set of vertices $V(G)$ and a finite set of undirected edges $E(G)$, where $V(G)$ and $E(G)$ are often denoted as $V$ and $E$, respectively. An edge $e$ incident upon two vertices $u, v$ in $G$ is denoted by $e=(u, v)$ unless any confusion arises. We denote $V(e)=\{u, v\}$, or generally $V(K)=\{u, v \in V \mid(u, v) \in K\}$ for a subset $K \subseteq E$. For disjoint sets $X, X^{\prime} \subset V$, we denote $\left(X, X^{\prime} ; G\right)=\left\{(u, v) \in E \mid u \in X\right.$ and $\left.v \in X^{\prime}\right\}$, where it is often written as $\left(X, X^{\prime}\right)$ if $G$ is clear from the context. We denote $d_{G}(X)=|(X, \bar{X} ; G)|$. This is called the degree of $X$ (in $\left.G\right)$. We set $d_{G}(S)=0$ if $S=\emptyset$. If $X=\{v\}$ then $d_{G}(\{v\})$ is denoted simply as $d_{G}(v)$ and is the total number of edges $\left(v, v^{\prime}\right), v^{\prime} \neq v$, incident upon $v$. We often denote $d_{G}(S)$ as $d(S)$ if $G$ is clear from the context. A path between vertices $u$ and $v$ is often called a $(u, v)$-path and denoted by $P_{G}(u, v)$, and is often written as $P(u, v)$ if $G$ is clear from the context. For two vertices $u, v$ of $G$, let $\lambda(u, v ; G)$, or simply $\lambda(u, v)$, denote the maximum number of pairwise edge-disjoint paths between $u$ and $v$.

For a set $X \subseteq V$, let $G[X]$ denote the subgraph having $X$ as its vertex set and $\{(u, v) \in E \mid u, v \in X\}$ as its edge set. $G[X]$ is called the subgraph of $G$ induced by $X$ (or the induced subgraph of $G$ by $X$ ). Deletion of $X \subseteq V$ from $G$ is to construct $G[V-X]$, which is often denoted as $G-X$. If $X=\{v\}$ then we often denote $G-v$ for simplicity. Deletion of $Q \subseteq E$ from $G$ defines a spanning subgraph of $G$, denoted by $G-Q$, having $E-Q$ as its edge set. If $Q=\{e\}$ then we denote $G-e$. For a set $E^{\prime}$ of edges such that $E^{\prime} \cap E=\emptyset$, let $G+E^{\prime}$ denote the graph $\left(V, E \cup E^{\prime}\right)$. If $E^{\prime}=\{e\}$ then we denote $G+e$.

Let $K \subseteq E$ be any minimal set such that $G-K$ has more components than $G$. $K$ is called a separator of $G$, or in particular a $(X, Y)$-separator if any vertex of $X$ and any one of $Y$ are disconnected in $G-K$. If $X=\{u\}$ or $Y=\{v\}$ then it is denoted as a $(u, Y)$-separator or a $(X, v)$-separator, respectively. A minimum $(X, Y)$-separator $K$ of $G$ is a $(X, Y)$-separator of minimum cardinality. Such 
$K$ is often called an $(X, Y)$-cut or an $|K|$-cut. It is known that a $(u, v)$-cut $K$ has $|K|=\lambda(u, v ; G)$. A minimum separator $K$ of $G$ is a separator of minimum cardinality among all separators of $G$, and $|K|$ is called the edge-connectivity (denoted by $\sigma$ ) of $G$; particularly we call such $K \subseteq E$ a minimum cut (of $G$ ). $G$ is said to be $k$-edge-connected if $\lambda(G) \geq k$. A $k$-edge-connected component (k-component, for short) of $G$ is a subset $S \subseteq V$ satisfying the following (a) and (b): (a) $\lambda(u, v ; G) \geq k$ for any pair $u, v \in S$; (b) $S$ is a maximal set that satisfies (a). Let $\Gamma_{G}(k)$ denote the set of all $k$-components of $G$. In a graph $G$ with $\lambda(G)=\sigma$, a $(\sigma+1)$-component $S$ with $d_{G}(S)=\sigma$ is called a leaf $(\sigma+1)$ component of $G$ (or a leaf of $G$, for short). It is known that $\lambda(G) \geq k$ if and only if $V$ is a $k$-component. Note that distinct $k$-components are disjoint sets. Each 1-component is often called a component.

Note that we assume that $|V| \geq \sigma+2$ in $(\sigma+1) \mathrm{ECA}(\mathrm{S}, \mathrm{SA})$, the subject of the paper.

A cactus is an undirected connected graph in which any pair of cycles share at most one vertex. A structural graph $F(G)$ of $G$ with $\lambda(G)=\sigma$ is a representation of all minimum cuts of $G$ and is introduced in [11. We use the term "nodes of $F(G)$ " to distinguish them from vertices of $G . F(G)$ is an edge-weighted cactus of $O(|V|)$ nodes and edges such that each tree edge (an edge which is a bridge in $F(G)$ ) has weight $\lambda(G)$ and each cycle edge (an edge included in any cycle) has weight $\lambda(G) / 2$. Let $F(G)$ be a structural graph of $G$. Particularly if $\sigma$ is odd then $F(G)$ is a weighted tree. (Examples of $G$ and $F(G)$ will be given in Figs. 1 and 2.) Each vertex in $G$ maps to exactly one node in $F(G)$, and $F(G)$ may have some other nodes, call empty nodes, to which no vertices of $G$ are mapped. Let $\epsilon(G) \subseteq V(F(G))$ denote the set of all empty nodes of $F(G)$. Note that any minimum cut of $G$ is represented as either a tree edge or a pair of two cycle edges in the same cycle of $F(G)$, and vice versa. Let $\rho: V \rightarrow V(F(G))-\epsilon(G)$ denote this mapping. We use the following notations: $\rho(X)=\{\rho(v) \mid v \in X\}$ for $X \subseteq V$, and $\rho^{-1}(Y)=\{v \in V \mid \rho(v) \in Y\}$ for $Y \subseteq V(F(G)) . \rho(\{v\})$ or $\rho^{-1}(\{v\})$ is written as $\rho(v)$ or $\rho^{-1}(v)$, respectively, for notational simplicity. For any cut $(X, V(F(G))-X ; F(G))$, if summation of weights of all edges contained in the cut is equal to $\sigma$ then $\left(\rho^{-1}(X), V-\rho^{-1}(X) ; G\right)$ is a $\sigma$-cut of $G$. Note that the cut of $F(G)$ consists of either one tree edge or a pair of two cycle edges in the same cycle of $F(G)$. Conversely, for any $\sigma$-cut $(X, V-X ; G), F(G)$ has at least one cut $(Y, V(F(G))-Y ; G)$ in which summation of weight of all edges contained in the cut is equal to $\sigma$, where $Y$ is a node set of $F(G)$ such that $\rho(X)=Y-\epsilon(G)$. Each $(\sigma+1)$-component $S$ of $G$ is represented as a vertex $\rho(S) \in V(F(G))-\epsilon(G)$ in $F(G)$, and, for any vertex $v \in V(F(G))-\epsilon(G), \rho^{-1}(v)$ is a $(\sigma+1)$-component of $G$. For $v \in V(F(G))$, if summation of weights of all edges that are incident to $v$ in $F(G)$ equals to $\sigma$, then $v$ is called a leaf node (that is a degree- 1 vertex in a tree or a degree- 2 vertex in a cycle). Note that, for any leaf node $v, \rho^{-1}(v)$ is a leaf of $G$, conversely, for any leaf $L$ of $G, \rho(L)$ is a leaf node of $F(G)$. It is shown that $F(G)$ can be constructed in $O(|V||E|)$ time [11] or in $O\left(\sigma^{2}|V| \log (|V| / \sigma)+|E|\right)$ time [7]. 
Two edges $e_{1}, e_{2}$ are said to be independent if and only if $V\left(e_{1}\right) \cap V\left(e_{2}\right)=\emptyset$, and a set $Q \subseteq E$ is called an independent set or a matching of $G$ if and only if any pair of edges in $Q$ are independent. An independent set of maximum cardinality in $G$ is called a maximum matching of $G$.

Proposition 1. [5] For distinct sets $X, Y \subset V$ of any graph $G=(V, E)$,

$$
\begin{array}{r}
d(X)+d(Y)=d(X-Y)+d(Y-X)+2|(V-X \cup Y, X \cap Y)| \\
d(X)+d(Y)=d(X \cap Y)+d(X \cup Y)+2|(X-Y, Y-X)| .
\end{array}
$$

Let $\lceil x\rceil(\lfloor x\rfloor$, respectively) denote the minimum integer no smaller (the maximum one no greater) than $x$.

\section{$2.2 \sigma$-Components and Leaf-Graphs}

Let $\lambda(G)=\sigma>0$. Let $X_{1}, X_{2}$ be distinct $(\sigma+1)$-components of $G$. The pair $\left\{X_{1}, X_{2}\right\}$ are called an adjacent pair (denoted as $X_{1} \chi X_{2}$ ) if any two vertices $w \in X_{1}$ and $w^{\prime} \in X_{2}$ are adjacent in $G$, or called a nonadjacent pair (denoted as $\left.X_{1} \bar{\chi} X_{2}\right)$ otherwise. Let

$$
V_{C}=\{v \mid v \text { represents an individual }(\sigma+1) \text {-component of } G\}
$$

and let $S(v) \in \Gamma_{G}(\sigma+1)$ denote the one represented by $v \in V_{C}$. Let $C(G)=$ $\left(V_{C}, E_{C}\right)$ be defined by $V_{C}$ and $E_{C}=\left\{\left(v, v^{\prime}\right) \mid v, v^{\prime} \in V_{C}\right.$ and $\left.S(v) \bar{\chi} S\left(v^{\prime}\right)\right\}$, and it is called the component graph of $G$. Let $L F(G)=\left\{X \in \Gamma_{G}(\sigma+1) \mid X\right.$ is a leaf of $\left.G\right\}$ and $V_{R}=\{v \mid v$ represents an individual leaf of $G\} \subseteq V_{C}$. Let $Y(v)$ denote the leaf $(\sigma+1)$-component represented by $v \in V_{R}$. Let $R(G)=\left(V_{R}, E_{R}\right)$ be the subgraph of $C(G)$ defined by $E_{R}=\left\{\left(v, v^{\prime}\right) \in E_{C} \mid v, v^{\prime} \in V_{R}\right.$ and $\left.Y(v) \bar{\chi} Y\left(v^{\prime}\right)\right\}$, and it is called the leaf-graph of $G$.

Property 1. $R(G)$ is simple.

Let $Y_{i}, i=1,2,3,4$, be distinct leaves of $G$. A set of two nonadjacent pairs $\left\{Y_{1}, Y_{2}\right\},\left\{Y_{3}, Y_{4}\right\}$ is called a $D$-combination if they are disjoint (that is, $\left\{Y_{1}, Y_{2}\right\} \cap$ $\left.\left\{Y_{3}, Y_{4}\right\}=\emptyset\right)$. In general, for $2 t$ distinct leaves $Y_{i}, i=1, \ldots, 2 t$, of $G$ with $t \geq 2$, a set of $t$ nonadjacent pairs $\left\{Y_{1}, Y_{2}\right\}, \ldots,\left\{Y_{2 t-1}, Y_{2 t}\right\}$ is called a D-set of $G$ if any two pairs of the set form a D-combination. Let $Y_{1} \chi\left\{Y_{2}, Y_{3}\right\}$ denote that both $Y_{1} \chi Y_{2}$ and $Y_{1} \chi Y_{3}$ hold. A D-combination $\left\{\left\{Y_{1}, Y_{2}\right\},\left\{Y_{3}, Y_{4}\right\}\right\}$ is called an $I$ combination (denoted as $\left\{Y_{1}, Y_{2}\right\} \angle\left\{Y_{3}, Y_{4}\right\}$ ) if either $Y_{1} \chi\left\{Y_{3}, Y_{4}\right\}$ or $Y_{2} \chi\left\{Y_{3}, Y_{4}\right\}$ holds. If neither $\left\{Y_{1}, Y_{2}\right\} \angle\left\{Y_{3}, Y_{4}\right\}$ nor $\left\{Y_{3}, Y_{4}\right\} \angle\left\{Y_{1}, Y_{2}\right\}$ holds then we denote $\left\{Y_{1}, Y_{2}\right\} \nmid\left\{Y_{3}, Y_{4}\right\}$.

We first show some basic results on $R(G)$ and leaves of $G$.

Proposition 2. Suppose that $G$ is simple. Then either $|Y|=1$ or $|Y| \geq \sigma+2$ for any $Y \in L F(G)$. 
Since each leaf $Y$ has $d_{G}(Y)=\sigma$, we obtain the next proposition by Proposition 2 .

Proposition 3. Suppose that $G$ is simple. If $\left\{Y_{1}, Y_{2}\right\} \subseteq L F(G)$ is an adjacent pair then $\left|Y_{1}\right|=\left|Y_{2}\right|=1$.

Proposition 4. $d_{R(G)}(v) \geq \max \left\{\left|V_{R}\right|-(\sigma+1), 0\right\}$ for any $v \in V_{R}$.

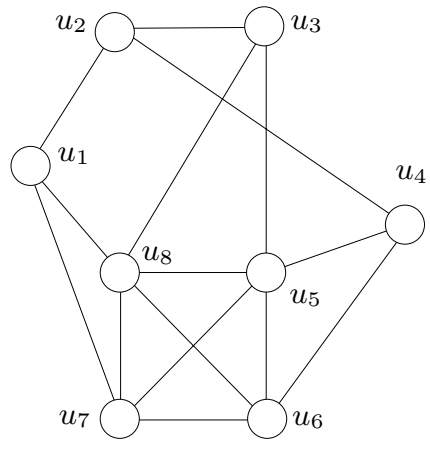

Fig. 1. A simple graph $G$ with $\lambda(G)=$ 3 and $|L F(G)|=4$.

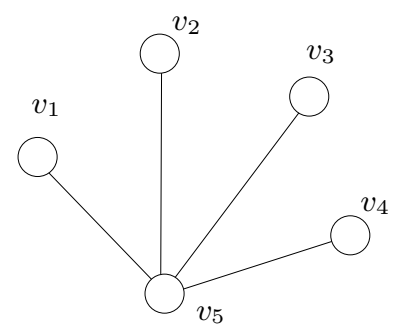

Fig. 2. A structural graph $F(G)$ of $G$ in Fig. 1 where all edge-weights are 3 and none of them are written. In this case leaves $Y_{i}$ in $L F(G)$ of the graph $G$ shown in Fig. 1 are represented as nodes $v_{i}$ of $F(G)$ for $i=1, \ldots, 5$ : it may happen that $G$ has a node to which no corresponding leaf of $L F(G)$ exists.

\section{$2.3 \quad$ Examples}

Let $G=(V, E)$ with $|V| \geq \sigma+2$ and $\lambda(G)=\sigma$ be any given simple graph. Let $O P T(M)$ or $O P T(S)$ denote the cardinality of an optimum solution to $(\sigma+1) \mathrm{ECA}(*, \mathrm{MA})$ or to $(\sigma+1) \mathrm{ECA}(\mathrm{S}, \mathrm{SA})$ for $G$, respectively. For $\sigma=3$, we give an example such that $O P T(S)=O P T(M)+1$. For the graph $G$ with $|L F(G)|=$ 4 shown Fig. 1, $R(G)$ is given in Fig. 3. The set of edges $\left\{\left(u_{1}, u_{3}\right),\left(u_{2}, u_{4}\right)\right\}$ is an optimum solution to $4 \mathrm{ECA}\left({ }^{*}, \mathrm{MA}\right)$, while $\left\{\left(u_{1}, u_{3}\right),\left(u_{2}, u_{8}\right),\left(u_{3}, u_{7}\right)\right\}$ is an optimum solution to $4 \mathrm{ECA}(\mathrm{S}, \mathrm{SA})$ and, therefore, $\operatorname{OPT}(S)=3=\operatorname{OPT}(M)+1$.

\section{Maximum Matchings of Leaf-Graphs}

One of requirements in finding a solution to $(\sigma+1) \mathrm{ECA}(\mathrm{S}, \mathrm{SA})$ or $(\sigma+1) \mathrm{ECA}(*$, $\mathrm{SA}$ ) with $\sigma \geq 1$ is to obtain a largest D-set. Hence, in this section, the cardinality of a maximum $\mathrm{D}$-set is investigated by considering a maximum matching $\mathcal{M}$ of $R(G)$. 


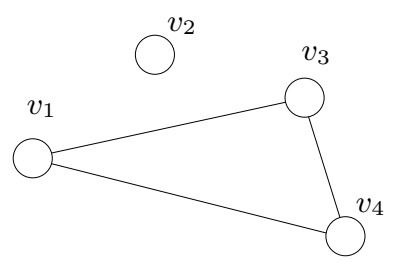

Fig. 3. The leaf-graph $R(G)$ of $G$ in Fig. 1]

Let $\mathcal{M}$ denote any fixed maximum matching of $R(G)$ in the following discussion unless otherwise stated, where we assume that $\lambda(G)=\sigma \geq 1$.

Proposition 5. $|\mathcal{M}|$ satisfies one of the following (1) (3).

(1) If $\left|V_{R}\right| \geq 2 \sigma+1$ or if $\sigma$ is even and $\left|V_{R}\right|=2 \sigma$ then $|\mathcal{M}|=\left\lfloor\left|V_{R}\right| / 2\right\rfloor$.

(2) If $\sigma$ is odd and $\left|V_{R}\right|=2 \sigma$ then

$$
\left\lfloor\left|V_{R}\right| / 2 \mid\right\rfloor-1 \leq|\mathcal{M}| \leq\left\lfloor\left|V_{R}\right| / 2\right\rfloor .
$$

(3) If $\left|V_{R}\right| \leq 2 \sigma-1$ then

$$
\max \left\{0, \min \left\{\left|V_{R}\right|-\sigma,\left\lfloor\left|V_{R}\right| / 2\right\rfloor\right\}\right\} \leq|\mathcal{M}| \leq\left\lfloor\left|V_{R}\right| / 2\right\rfloor .
$$

Corollary 1. Suppose that $\left|V_{R}\right|=2 \sigma$ and $\sigma=2 m+1$. If $|\mathcal{M}|=\left\lfloor\left|V_{R}\right| / 2\right\rfloor-1$ then $G=(V, E)$ is a complete bipartite graph with $V=X \cup Y, X \cap Y=\emptyset$, $|X|=|Y|=\sigma$ and $E=\{(x, y) \mid x \in X, y \in Y\}$.

The relationship among $G, C(G)$ and $R(G)$ shows the following proposition concerning $\left|V_{R}\right|,|\mathcal{M}|$ and $\left|E^{\prime}\right|$ of any optimum solution $E^{\prime}$ to $(\sigma+1) \operatorname{ECA}(\mathrm{S}, \mathrm{SA})$.

Proposition 6. Let $E^{\prime}$ be any solution to $G$ in $(\sigma+1) E C A(S, S A)$ and $\mathcal{M}$ be a maximum matching of $R(G)$. Then

$$
\left|V_{R}\right|-|\mathcal{M}| \leq\left|E^{\prime}\right|
$$

\section{Augmentation by Edge-Interchange}

We explain an operation called edge-interchange which was originally introduced in 20 21] for an efficient augmentation. It is also used in [14151617]18]. Let $L F(G)=\left\{Y_{1}, \ldots, Y_{q}\right\}(q=|L F(G)|)$ denote the class of all leaves of $G$ and choose $y_{i} \in Y_{i}$ as the representative of $Y_{i}$. Let

$$
Y(G)=\left\{y_{i} \mid Y_{i} \in L F(G)\right\}, \quad q \geq 2, \text { and } r=\lceil q / 2\rceil .
$$

We can easily prove the next proposition.

Proposition 7. If there is a set $E^{\prime}$ of edges, each connecting vertices of $G$, such that $E^{\prime} \cap E=\emptyset$ and $V\left(E^{\prime}\right)=Y(G) \subseteq S$ for some $(\sigma+1)$-component $S$ of $G+E^{\prime}$, then $S=V$.

Let $Y$ stand for $Y(G)$ in the rest of the section. 


\subsection{Attachments}

We have $d_{G}\left(Y_{i}\right)=\sigma$ and $\lambda\left(y_{i}, y_{j} ; G\right)=\sigma$ for any $y_{i}, y_{j} \in Y(i \neq j)$. An edge set $F$ is called an attachment (for $G$ ) if and only if the following (1) through (4) hold:

(1) $V(F) \subseteq Y$,

(2) $F \cap E(G)=\emptyset$,

(3) $V(e) \neq V\left(e^{\prime}\right)\left(\forall e, e^{\prime} \in F, e \neq e^{\prime}\right)$, and

(4) if $q(=|L F(G)|)$ is odd then $F$ has at most one pair $f, f^{\prime}$ such that $\mid V(f) \cap$ $V\left(f^{\prime}\right) \mid=1$; or if $q$ is even then $F$ has no such pair.

Let $F$ be any attachment for $G$. For each $e=(u, v) \in F, G+F$ has a new $(\sigma+1)$-component, denoted by $\mathcal{A}(e, G+F)$, containing $V(e)$.

We are going to show that we can find a minimum attachment $Z(\sigma+1)=$ $\left\{e_{1}, \ldots, e_{r}\right\}(r=\lceil q / 2\rceil)$ such that $\lambda(G+Z(\sigma+1))=\sigma+1$. Although there are two cases: $r=1$ and $r \geq 2$, we discuss only the latter case in the following. (Note that if $r=1$ then we immediately obtain the desired attachment $F$.)

\subsection{Finding a Minimum Attachment}

Suppose that there are an attachment $F$ for $G$ and vertices $y_{i j} \in Y-V(F)$, $1 \leq i, j \leq 2$, where $y_{11}, y_{12}, y_{21}$ are distinct, and if $y_{22}$ is equal to one of the other three then we assume that $y_{22}=y_{21}$ (see Fig. 44). We use the following

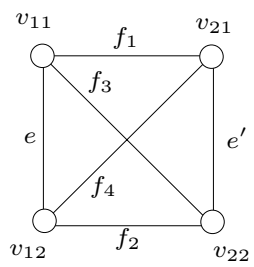

(1)

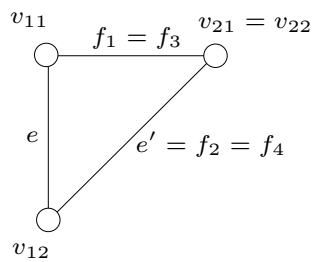

$(2)$

Fig. 4. The edges $e, e^{\prime}$ and $f_{i}, 1 \leq i \leq 4$ : (1) $y_{21} \neq y_{22}$; (2) $y_{21}=y_{22}$.

notations:

$$
\begin{gathered}
L=G+F, \quad e=\left(y_{11}, y_{12}\right), \quad e^{\prime}= \begin{cases}\left(y_{21}, y_{22}\right) & \text { if } y_{21} \neq y_{22} \\
\left(y_{12}, y_{21}\right) & \text { if } y_{21}=y_{22},\end{cases} \\
\mathcal{A}(e)=\mathcal{A}\left(e, L+\left\{e, e^{\prime}\right\}\right), \quad \mathcal{A}\left(e^{\prime}\right)=\mathcal{A}\left(e^{\prime}, L+\left\{e, e^{\prime}\right\}\right), \\
f_{1}=\left(y_{11}, y_{21}\right), \quad f_{2}=\left(y_{12}, y_{22}\right), \quad f_{3}=\left(y_{11}, y_{22}\right), \quad f_{4}=\left(y_{12}, y_{21}\right),
\end{gathered}
$$


where we set $f_{1}=f_{3}$ and $e^{\prime}=f_{2}=f_{4}$ if $y_{21}=y_{22}$, and

$$
\mathcal{A}\left(f_{i}\right)= \begin{cases}\mathcal{A}\left(f_{i}, L+\left\{f_{1}, f_{2}\right\}\right) & \text { if } 1 \leq i \leq 2 \\ \mathcal{A}\left(f_{i}, L+\left\{f_{3}, f_{4}\right\}\right) & \text { if } 3 \leq i \leq 4\end{cases}
$$

Note that $e, e^{\prime}, f_{i} \notin E(L), 1 \leq i \leq 4$. We have the following two cases.

Case I: $\mathcal{A}(e) \cap \mathcal{A}\left(e^{\prime}\right)=\emptyset$; Case II: $\mathcal{A}(e) \cap \mathcal{A}\left(e^{\prime}\right) \neq \emptyset$ (that is, $\left.\mathcal{A}(e)=\mathcal{A}\left(e^{\prime}\right)\right)$.

For Case I, we are going to show that there are two edges $f, f^{\prime}$, with $V(f) \cup$ $V\left(f^{\prime}\right)=V(e) \cup V\left(e^{\prime}\right)$, such that

$$
\mathcal{A}(e) \cup \mathcal{A}\left(e^{\prime}\right) \subseteq \mathcal{A}\left(f, L+\left\{f, f^{\prime}\right\}\right)=\mathcal{A}\left(f^{\prime}, L+\left\{f, f^{\prime}\right\}\right) .
$$

That is, we can add two edges so that one $(\sigma+1)$-component containing $\mathcal{A}(e) \cup$ $\mathcal{A}\left(e^{\prime}\right)$ may be obtained. Finding and adding such a pair of edges $f, f^{\prime}$ is called edge-interchange (with respect to $V\left(e_{1}\right) \cup V\left(e_{2}\right)$ ).

Suppose that $\mathcal{A}(e) \cap \mathcal{A}\left(e^{\prime}\right)=\emptyset$. Note that $y_{21} \neq y_{22}$ in this case. Let $K$ be any fixed $\left(\mathcal{A}(e), \mathcal{A}\left(e^{\prime}\right)\right)$-cut of $L+\left\{e, e^{\prime}\right\}$, and let $B_{i}, 1 \leq i \leq 2$, denote the two sets of vertices in $L+\left\{e, e^{\prime}\right\}$ such that $B_{1} \cup B_{2}=V, B_{2}=V-B_{1}$, $K=\left(B_{1}, B_{2} ; L+\left\{e, e^{\prime}\right\}\right), \mathcal{A}(e) \subseteq B_{1}$ and $\mathcal{A}\left(e^{\prime}\right) \subseteq B_{2} \cdot|K|=\sigma=\lambda\left(y_{1}, y_{2} ; L^{\prime \prime}\right)$ for any $y_{i} \in B_{i}, 1 \leq i \leq 2$, where $L^{\prime \prime}$ denotes $L, L+e, L+e^{\prime}$ or $L+\left\{e, e^{\prime}\right\}$. $K$ is a $\left(y_{1}, y_{2}\right)$-cut of $L$. Suppose that $f$ and $f^{\prime}$ satisfy either (i) or (ii):

(i) $f=f_{1}, f^{\prime}=f_{2}$, or (ii) $f=f_{3}, f^{\prime}=f_{4}$,

where $\left\{f, f^{\prime}\right\} \cap E(L)=\emptyset$.

The next proposition shows a property of edge-interchange.

Proposition 8. If $\mathcal{A}(e) \cap \mathcal{A}\left(e^{\prime}\right)=\mathcal{A}\left(f_{1}\right) \cap \mathcal{A}\left(f_{2}\right)=\emptyset$ then $\mathcal{A}\left(f_{3}\right) \cap \mathcal{A}\left(f_{4}\right) \neq \emptyset$, that is, $\mathcal{A}\left(f_{3}\right)=\mathcal{A}\left(f_{4}\right)$.

Let $\left\{f, f^{\prime}\right\}$ denote the following pair of edges:

$$
\begin{gathered}
\left\{e, e^{\prime}\right\} \text { if } \mathcal{A}(e)=\mathcal{A}\left(e^{\prime}\right) \text { (the case with } V(e) \cap V\left(e^{\prime}\right)=\emptyset \text { is included); } \\
\left\{f_{1}, f_{2}\right\} \text { if } \mathcal{A}(e) \cap \mathcal{A}\left(e^{\prime}\right)=\emptyset \text { and } \mathcal{A}\left(f_{1}\right)=\mathcal{A}\left(f_{2}\right) ; \\
\left\{f_{3}, f_{4}\right\} \text { if } \mathcal{A}(e) \cap \mathcal{A}\left(e^{\prime}\right)=\mathcal{A}\left(f_{1}\right) \cap \mathcal{A}\left(f_{2}\right)=\emptyset .
\end{gathered}
$$

Clearly, $\left\{f, f^{\prime}\right\} \cap E(L)=\emptyset$. Such a pair $f, f^{\prime}$ are called an augmenting pair (with respect to $\left.\left\{y_{11}, y_{12}, y_{21}, y_{22}\right\}\right)$ of $L$.

Corollary 2. Let $L^{\prime}=L+\left\{f, f^{\prime}\right\}$ for any augmenting pair $f, f^{\prime}$. Then $L^{\prime}-f^{\prime}$ has no $\sigma$-cut separating $V\left(f^{\prime}\right)$ from $V(f)$. That is, if $L^{\prime}-f^{\prime}$ has a $\sigma$-cut $K$ separating a vertex of $V\left(f^{\prime}\right)$ from $V(f)$ then $K$ separates the two vertices of $V\left(f^{\prime}\right)$.

From Corollary 2, other important properties (Proposition 911) of edgeinterchange are obtained. 

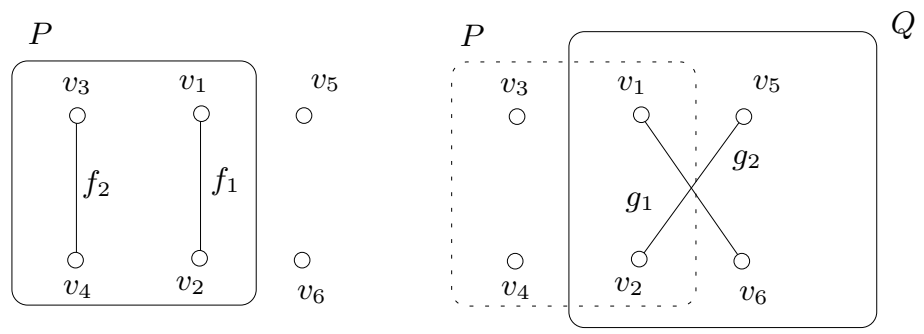

Fig. 5. The two $(\sigma+1)$-components $\mathcal{A}\left(f_{1}, G+\left\{f_{1}, f_{2}\right\}\right)$ and $\mathcal{A}\left(g_{1}, G+\left\{g_{1}, g_{2}\right\}\right)$ produced by two augmenting pairs $\left\{f_{1}, f_{2}\right\}$ and $\left\{g_{1}, g_{2}\right\}$, respectively.

Proposition 9. Suppose that $G$ has six leaves $Y_{i} \in L F(G)(1 \leq i \leq 6)$, and choose $y_{i} \in Y_{i}$ as a representative of each $Y_{i}$. Suppose that $\left\{f_{1}, f_{2}\right\}$ is an augmenting pair with respect to $\left\{y_{i} \mid 1 \leq i \leq 4\right\}$ of $G$. If $\mathcal{A}\left(f_{1}, G+\left\{f_{1}, f_{2}\right\}\right)$ is a leaf then, for each $i \in\{1,2\}$, there is an augmenting pair $\left\{g_{1}, g_{2}\right\}$ with respect to $V\left(f_{i}\right) \cup\left\{y_{5}, y_{6}\right\}$ of $G$ such that $\mathcal{A}\left(g_{1}, G+\left\{g_{1}, g_{2}\right\}\right)$ is not a leaf (see Fig. 5).

By Proposition 9, we obtain the following procedure that is a modified version of the procedure given in [15]. It finds a sequence of edges $e_{1}, \ldots, e_{r}$ $(r=\lceil|L F(G)| / 2\rceil \geq 1)$ by repeating edge-interchange operation, where handling the case with $|L F(G)|=2$ is included. Note that edges with which we are concerned are those connecting vertices belonging to distinct leaves. If an edge $g$ connects a vertex in a leaf $Y_{i}$ and another vertex in a leaf $Y_{j}(i \neq j)$ then, for simplicity, we say that $g$ connects $Y_{i}$ and $Y_{j}$.

\section{Procedure FIND_EDGES;}

begin

1. $G_{1} \leftarrow G ; \pi \leftarrow L F(G) ; i \leftarrow 1 ; E_{1}^{\prime} \leftarrow \emptyset$;

2. while $\pi \neq \emptyset$ do

begin

3. if $|\pi|=2$ then

4. $\quad f_{i} \leftarrow$ an edge connecting the two leaves of $\pi ; E_{i}^{\prime \prime} \leftarrow\left\{f_{i}\right\}$;

5. $\quad$ else if $|\pi| \leq 5$ then

6. $\quad$ Find an augmenting pair $E_{i}^{\prime \prime}=\left\{f_{i}, f_{i}^{\prime}\right\}$ by Proposition 8

7. $\quad$ else $/ *|\pi| \geq 6 * /$

8. $\quad$ Find an augmenting pair $E_{i}^{\prime \prime}=\left\{f_{i}, f_{i}^{\prime}\right\}$ by Proposition 9 ,

9. $\quad E_{i+1}^{\prime} \leftarrow E_{i}^{\prime} \cup E_{i}^{\prime \prime} ; G_{i+1} \leftarrow G_{i}+E_{i}^{\prime \prime} ; \pi \leftarrow \pi-\left\{Y(v) \mid v \in V\left(E_{i}^{\prime \prime}\right)\right\} ; i \leftarrow i+1 ;$ end

end;

Proposition 10. $G_{i+1}$ has a leaf containing $\mathcal{A}\left(f_{i}, G_{i+1}\right)$ if and only if $\left|L F\left(G_{i}\right)\right|$ $=5$ just after the execution of Step 9 in FIND_EDGES. 
Note that executing Step 6 or Step 8 once can be done in $O\left(\left|V_{R}\right|\right)$ by using a structural graph $F(G)$, and we can construct $F(G)$ in $O\left(\sigma^{2}|V| \log (|V| / \sigma)+|E|\right)$ time (see 7]). The details are omitted here.

The next proposition holds for the edge set $E^{\prime}$ produced by FIND_EDGES.

Proposition 11. Let $Z(\sigma+1)=\left\{e_{1}, \ldots, e_{r}\right\}(r=\lfloor\mid L F(G) / 2\rfloor)$ be given by FIND_EDGES. Then $Z(\sigma+1)$ is a minimum attachment such that $\lambda\left(G^{\prime}\right)=\sigma+1$, where $G^{\prime}=G+Z(\sigma+1)$. Furthermore the procedure runs in $O\left(\sigma^{2}|V| \log (|V| / \sigma)+\right.$ $\left.|E|+\left|V_{R}\right|^{2}\right)$ time.

\section{$5(\sigma+1) \operatorname{ECA}(\mathrm{S}, \mathrm{SA})$ for $G$ Having Less Than $2 \sigma+6$ Leaves}

We denote $L F(G)=\left\{Y_{i} \mid 1 \leq i \leq q\right\}(q=|L F(G)|), Y(G)=\left\{y_{1}, \ldots, y_{q}\right\}$ and $V_{R}=\left\{v_{1}, \ldots, v_{q}\right\}$, where each $y_{i}$ is represented as $v_{i}$ in $R(G)$. First we consider the case where $G$ has two or three leaves.

Proposition 12. If $q=2$ then the following (1) or (2) holds.

(1) If $Y_{1} \bar{\chi} Y_{2}$ then $|\mathcal{M}|=1$, there are two vertices $y_{i} \in Y_{i}, i=1,2$, such that $E^{\prime}=\left\{\left(y_{1}, y_{2}\right)\right\}$ is a solution, and $\operatorname{OPT}(S)=O P T(M)=1$.

(2) If $Y_{1} \chi Y_{2}$ then $|\mathcal{M}|=0$, there are three vertices $y_{i} \in Y_{i}(i=1,2), x \in$ $V-\left(Y_{1} \cup Y_{2}\right)$ such that $E^{\prime}=\left\{\left(y_{1}, x\right),\left(y_{2}, x\right)\right\}$ is a solution, and $\operatorname{OPT}(S)=$ $2=O P T(M)+1$.

Proposition 13. If $q=3$ and there exist two leaves $Y_{1}, Y_{2}$ with $Y_{1} \bar{\chi} Y_{2}$ then $|\mathcal{M}|=1$, there are distinct edges $e_{1}, e_{2}$ such that $E^{\prime}=\left\{e_{1}, e_{2}\right\}$ is a solution, and $O P T(S)=O P T(M)=2$.

Next we consider the remaining case where $3 \leq q<2 \sigma+6$. For each $e^{\prime}=$ $\left(x^{\prime}, y^{\prime}\right) \in \mathcal{M}$, we can choose two vertices $x \in Y\left(x^{\prime}\right), y \in Y\left(y^{\prime}\right)$, and let $e=(x, y)$ be an edge, which is not included in $E$. We fix such an edge $e$ for each $e^{\prime} \in \mathcal{M}$, and let

$$
E_{0}^{\prime}=\left\{e=(x, y) \mid\left(x^{\prime}, y^{\prime}\right) \in \mathcal{M}\right\}
$$

Proposition 14. $\left|E_{0}^{\prime}\right|=|\mathcal{M}|$ and $E_{0}^{\prime} \cap E=\emptyset$.

In the rest of this section, we consider the graph $G+E_{0}^{\prime}$. First we define two sets $\mathcal{L}_{1}$ and $\mathcal{K}_{1}$ as follows.

Let $G_{1}=G+E_{0}^{\prime}$ and let $\mathcal{L}_{1}$ be the set of new leaves of $G_{1}$ created by adding $E_{0}^{\prime}$ to $G$. Clearly $\left|\mathcal{L}_{1}\right| \leq|\mathcal{M}|$. Let $\mathcal{K}_{1}=L F\left(G+E_{0}^{\prime}\right)-\mathcal{L}_{1}(\subseteq L F(G))$. Since $\mathcal{M}$ is a maximum matching of $R(G)$, Proposition 3 shows that each leaf in $\mathcal{K}_{1}$ consists of only one vertex and that the set of vertices $\mathcal{K}_{1}^{\prime}=\left\{x \mid\{x\} \in \mathcal{K}_{1}\right\}$ induces a complete graph of $G$ and of $G+E_{0}^{\prime}$.

We are going to propose an $O\left(\sigma^{2}|V| \log (|V| / \sigma)+|E|+\left|V_{R}\right|^{2}\right)$ time algorithm such that it finds an optimum solution if $\left|\mathcal{L}_{1}\right| \leq\left|\mathcal{K}_{1}\right|$ and such that a $\frac{3}{2}$-approximate solution if $\left|\mathcal{L}_{1}\right|>\left|\mathcal{K}_{1}\right|$. Note that we have $\left|\mathcal{L}_{1}\right| \leq\left|\mathcal{K}_{1}\right|$ if $|\mathcal{M}| \leq\left\lfloor\left|V_{R}\right| / 3\right\rfloor$. 
Proposition 15. Let $\left\{y_{1}^{\prime}\right\},\left\{y_{2}^{\prime}\right\} \in \mathcal{K}_{1}\left(y_{1}^{\prime} \neq y_{2}^{\prime}\right)$ and $Y_{1}, Y_{2} \in \mathcal{L}_{1}\left(Y_{1} \neq Y_{2}\right)$. If $\left\{\left(y_{1}, y_{1}^{\prime}\right),\left(y_{2}, y_{2}^{\prime}\right)\right\}$ is not an augmenting pair with $y_{1} \in Y_{1}$ and $y_{2} \in Y_{2}$ then there are $y_{3} \in Y_{1}$ and $y_{4} \in Y_{2}$ such that $\left\{\left(y_{4}, y_{1}^{\prime}\right),\left(y_{3}, y_{2}^{\prime}\right)\right\}$ is an augmenting pair and $\left(y_{4}, y_{1}^{\prime}\right),\left(y_{3}, y_{2}^{\prime}\right) \notin E$ (See Fig. G).
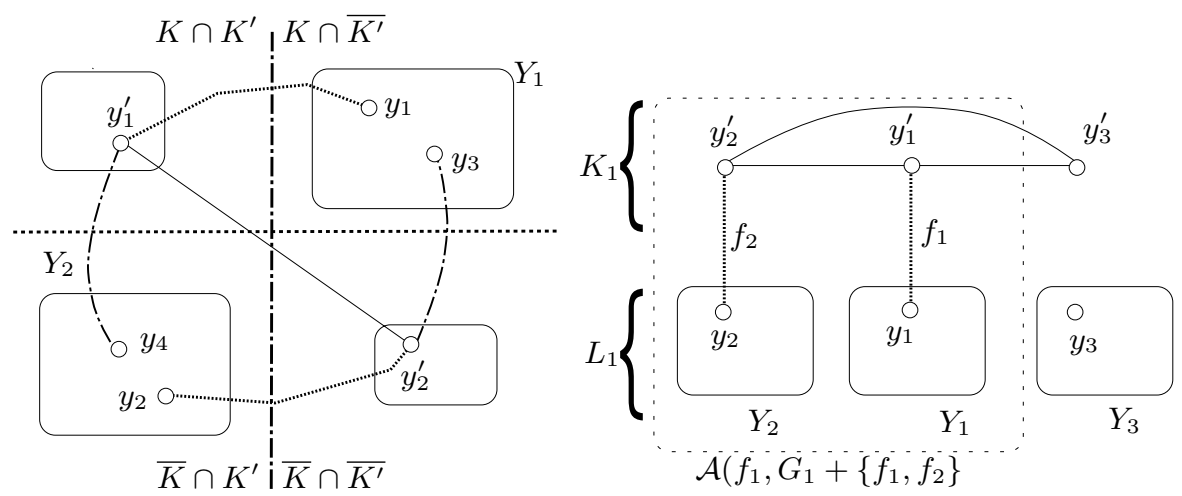

Fig. 6. A situation for Proposition 15

Fig. 7. $\mathcal{A}\left(f_{1}, G+\left\{f_{1}, f_{2}\right\}\right)$ in the proof of Proposition 16

We obtain the next proposition by Propositions 9 and 15

Proposition 16. Assume that $\left|\mathcal{L}_{1}\right| \geq 3$ and $\left|\mathcal{K}_{1}\right| \geq 3$. Then there exists an augmenting pair $\left\{f_{1}, f_{2}\right\}$ such that $f_{1}=\left(y_{1}, y_{1}^{\prime}\right) \notin E \cup E_{0}^{\prime}, f_{2}=\left(y_{2}, y_{2}^{\prime}\right) \notin E \cup E_{0}^{\prime}$, $\left\{\left\{y_{1}^{\prime}\right\},\left\{y_{2}^{\prime}\right\}\right\} \subseteq \mathcal{K}_{1}\left(y_{1}^{\prime} \neq y_{2}^{\prime}\right), \mathcal{L}_{1}$ has two distinct sets $Y_{1}, Y_{2}$ with $y_{1} \in Y_{1}, y_{2} \in Y_{2}$ and $\mathcal{A}\left(f_{1}, G+\left\{f_{1}, f_{2}\right\}\right)$ is not a leaf. Furthermore $\left.\mathcal{L}_{1} \cup \mathcal{K}_{1}-\left\{\left\{y_{1}^{\prime}\right\},\left\{y_{2}^{\prime}\right\}\right\}, Y_{1}, Y_{2}\right\}$ is the set of all leaves in $G_{1}+\left\{f_{1}, f_{2}\right\}$. (See Fig. 7)

Next we are going to discuss the case where $\left|\mathcal{L}_{1}\right| \leq 2$ or $\left|\mathcal{K}_{1}\right| \leq 2$.

Proposition 17. Suppose that $\left|\mathcal{L}_{1}\right| \leq 2$ and $\left|\mathcal{L}_{1}\right| \leq\left|\mathcal{K}_{1}\right|$. Then there exists a set $E_{2}^{\prime}=\left\{f_{1}, \ldots, f_{\left|\mathcal{K}_{1}\right|}\right\}$ such that $\lambda\left(G_{1}+E_{2}^{\prime}\right) \geq \sigma+1$ and $E_{2}^{\prime} \cap\left(E \cup E_{0}^{\prime}\right)=\emptyset$.

It remains to consider the cases $\left(\left|\mathcal{L}_{1}\right| \geq 3\right.$ and $\left.\left|\mathcal{K}_{1}\right| \leq 2\right)$ and $\left(\left|\mathcal{L}_{1}\right| \leq 2\right.$ and $\left.\left|\mathcal{L}_{1}\right|>\left|\mathcal{K}_{1}\right|\right)$, for which the next proposition holds.

Proposition 18. Suppose that one of the following (1)-(3) holds: (1) $\left|\mathcal{L}_{1}\right| \geq 3$ and $\left|\mathcal{K}_{1}\right| \leq 2$; (2) $\left|\mathcal{L}_{1}\right|=2$ and $\left|\mathcal{K}_{1}\right|=1$; (3) $\left|\mathcal{L}_{1}\right|=2$ and $\left|\mathcal{K}_{1}\right|=0$. Let $q_{1}=\left|L F\left(G_{1}\right)\right|$ and $r_{1}=\left\lceil\frac{q_{1}}{2}\right\rceil$. Then there exists a set $E_{2}^{\prime \prime}=\left\{f_{1}, \ldots, f_{r_{1}}\right\}$ such that $\lambda\left(G_{1}+E_{2}^{\prime \prime}\right) \geq \sigma+1$ and $E_{2}^{\prime \prime} \cap\left(E \cup E_{0}^{\prime}\right)=\emptyset$.

The discussion from Propositions 16 through 18 is summarized in the following procedure FIND_EDGES2. 


\section{Procedure FIND_EDGES2;}

begin

1. $G_{0} \leftarrow G ; \pi \leftarrow L F(G) ; E_{0}^{\prime} \leftarrow \emptyset ; \rho \leftarrow \emptyset$;

2. Find an edge set $E_{0}^{\prime}$ as in Proposition 14, $G_{1} \leftarrow G_{0}+E_{0}^{\prime}$;

Determine $\mathcal{L}_{1}$ and $\mathcal{K}_{1} ; i \leftarrow 1$;

3. while $\mathcal{K}_{i} \neq \emptyset$ do

begin

4. $\quad$ if $\left|\mathcal{L}_{i}\right| \geq 3$ and $\left|\mathcal{K}_{i}\right| \geq 3$ then

Find an augmenting pair $\left\{f, f^{\prime}\right\}$ by Proposition 16 $E_{i}^{\prime \prime} \leftarrow\left\{f, f^{\prime}\right\}$;

5. $\quad$ else if $\left|\mathcal{L}_{i}\right| \leq 2$ and $\left|\mathcal{L}_{i}\right| \leq\left|\mathcal{K}_{i}\right|$ then

Find an edge set $E_{i}^{\prime \prime}$ by Proposition [17,

6. else

Find an edge set $E_{i}^{\prime \prime}$ by Proposition 18 ;

7. $\quad$ Construct $\mathcal{K}_{i+1}$ and $\mathcal{L}_{i+1} ; E_{i}^{\prime} \leftarrow E_{i-1}^{\prime} \cup E_{i}^{\prime \prime} ; G_{i+1} \leftarrow G_{i}+E_{i}^{\prime \prime} ; i \leftarrow i+1$; end;

8. if $\lambda\left(G_{i}\right)=\sigma$ then $/ *$ the case with $\left|\mathcal{L}_{i}\right| \neq 0 * /$ end;

Find an edge set $E_{i}^{\prime \prime}$ by Proposition 18 ; $E_{i+1}^{\prime} \leftarrow E_{i-1}^{\prime} \cup E_{i}^{\prime \prime}$;

Proposition 19. FIND_EDGES2 produces an optimum solution if $\left|\mathcal{L}_{1}\right| \leq\left|\mathcal{K}_{1}\right|$.

Proposition 20. FIND_EDGES2 gives a $\frac{3}{2}$-approximate solution if $\left|\mathcal{L}_{1}\right|>\left|\mathcal{K}_{1}\right|$.

Remark 1. Let $\mathcal{M}$ be any maximum matching of $R(G)$. If $|\mathcal{M}| \leq\left\lfloor\frac{|L F(G)|}{3}\right\rfloor$ then $\left|\mathcal{L}_{1}\right| \leq\left|\mathcal{K}_{1}\right|$ and we can find an optimum solution in polynomial time. If $\left\lfloor\frac{|L F(G)|}{3}\right\rfloor<|\mathcal{M}| \leq\left\lfloor\frac{|L F(G)|}{2}\right\rfloor$ then $\left|\mathcal{L}_{1}\right| \leq\left|\mathcal{K}_{1}\right|$ or $\left|\mathcal{L}_{1}\right|>\left|\mathcal{K}_{1}\right|$. Since the proof of $\mathrm{NP}$-completeness of $k \mathrm{ECA}(\mathrm{S}, \mathrm{SA})$ in [10] is given for the case with $|\mathcal{M}|=$ $\left\lfloor\frac{|L F(G)|}{2}\right\rfloor$, we consider approximate solutions if $\left|\mathcal{L}_{1}\right|>\left|\mathcal{K}_{1}\right|$.

Theorem 1. Suppose that $|L F(G)| \leq 2 \sigma+6$. Then FIND_EDGES2 can find an optimum solution if $\left|\mathcal{L}_{1}\right| \leq\left|\mathcal{K}_{1}\right|$, or a $\frac{3}{2}$-approximate solution if $\left|\mathcal{L}_{1}\right|>\left|\mathcal{K}_{1}\right|$, in $O\left(\sigma^{2}|V| \log (|V| / \sigma)+|E|\right)$ time.

\section{$6(\sigma+1) \operatorname{ECA}(\mathrm{S}, \mathrm{SA})$ for $G$ Having at Least $2 \sigma+6$ Leaves}

In this case, Proposition [3) shows that any maximum matching $\mathcal{M}$ of $R(G)$ has $|\mathcal{M}|=\left\lfloor\frac{|L F(G)|}{2}\right\rfloor$. First, some basic results on nonadjacent pairs and edge interchange operation are going to be given.

Proposition 21. Suppose that there are a nonadjacent pair of leaves $Y_{1}, Y_{2} \in$ $L F(G)$ and two vertices $y_{i} \in Y_{i}, i=1,2$, with $\left(y_{1}, y_{2}\right) \notin E$, such that $G^{\prime}=$ $G+\left\{\left(y_{1}, y_{2}\right)\right\}$ has a leaf $S$ containing $Y_{1} \cup Y_{2}$. Let $\mathcal{L}^{\prime}=\left\{Y \subseteq S \mid Y \in \Gamma_{G}(\sigma+1)\right\}$, $X=Y_{1} \cup Y_{2}$ and $Z=\bigcup_{Y \in L F(G)-\left\{Y_{1}, Y_{2}\right\}} Y$. Then $|(X, Z ; G)| \leq \sigma-1$ if $\left|\mathcal{L}^{\prime}\right| \geq 3$. 
The next proposition can be proved by using Propositon 21 .

Proposition 22. Suppose $\sigma \geq 3$ and let $\mathcal{M}^{\prime}=\left\{\left(v_{2 i-1}, v_{2 i}\right) \mid 1 \leq i \leq m\right\} \subseteq \mathcal{M}$ for some $m \leq|\mathcal{M}|$, and put $Y_{j}=Y\left(v_{j}\right)$ for each $v_{j} \in V_{R}$.

(1) If $\left|\mathcal{M}^{\prime}\right| \geq 2$ and there are distinct indices $i, j$ with $1 \leq i, j \leq m$ such that $\left\{Y_{2 i-1}, Y_{2 i}\right\} \Varangle\left\{Y_{2 j-1}, Y_{2 j}\right\}$ then (i) and (ii) hold.

(i) These leaves are partitioned into a D-combination $\left\{\left\{L_{1}^{\prime}, L_{2}^{\prime}\right\},\left\{L_{3}^{\prime}, L_{4}^{\prime}\right\}\right\}$ having four vertices $y_{t} \in L_{t}^{\prime}, t=1,2,3,4$, such that $G+\left\{\left(y_{1}, y_{2}\right),\left(y_{3}, y_{4}\right)\right\}$ has a $(\sigma+1)$-component $S$ containing all $L_{t}^{\prime}, t=1,2,3,4$.

(ii) The $(\sigma+1)$-component $S^{\prime}$ of $G+\left\{\left(y_{1}, y_{2}\right)\right\}$ such that $L_{1}^{\prime} \cup L_{2}^{\prime} \subseteq S^{\prime}$ is not a leaf.

(2) If $\left|\mathcal{M}^{\prime}\right| \geq\lceil\sigma / 2\rceil+1$ and no such pair of indices as in (1) exist then, for each $\left(v_{2 i-1}, v_{2 i}\right) \in \mathcal{M}^{\prime}$, there are vertices $y_{2 i-1} \in Y_{2 i-1}$ and $y_{2 i} \in Y_{2 i}$ such that $G^{\prime}=G+\left\{\left(y_{2 i-1}, y_{2 i}\right)\right\}$ is a simple graph having a $(\sigma+1)$-component $X$ which is not a leaf and which contains $Y_{2 i-1} \cup Y_{2 i}$.

Proposition 23. Suppose that there is a set $\mathcal{M}^{\prime}=\left\{\left(v_{2 i-1}, v_{2 i}\right) \mid 1 \leq i \leq m\right\} \subseteq$ $\mathcal{M}$ for some $m$ with $\sigma+2 \leq m \leq|\mathcal{M}|$, and put $Y_{i}=Y\left(v_{i}\right)$ for each $v_{i} \in V_{R}$. Then there is an edge $\left(v_{2 h-1}, v_{2 h}\right) \in \mathcal{M}^{\prime}$ with $\left\{Y_{1}, Y_{2}\right\} \nless\left\{Y_{2 h-1}, Y_{2 h}\right\}$.

By combining Propositions 9, 22, and 23, we obtain the following proposition.

Proposition 24. Suppose that there is a set $\mathcal{M}^{\prime}=\left\{f_{i}=\left(v_{2 i-1}, v_{2 i}\right) \mid 1 \leq i \leq\right.$ $m\} \subseteq \mathcal{M}$ for some $m$ with $\sigma+3 \leq m \leq|\mathcal{M}|$, and put $Y_{i}=Y\left(v_{i}\right)$ for each $v_{i} \in V_{R}$. Then there exists an augmenting pair $\left\{e_{1}^{\prime}, e_{2}^{\prime}\right\}$ with respect to $Y_{1}, Y_{2}, Y_{2 j-1}, Y_{2 j}$ such that $G+\left\{e_{1}^{\prime}, e_{2}^{\prime}\right\}$ is simple and has no leaf $S$ with $Y_{1} \cup Y_{2} \cup Y_{2 j-1} \cup Y_{2 j} \subseteq S$, where $\left\{f_{1}, f_{j}\right\} \subseteq \mathcal{M}^{\prime}$.

Based on Proposition 24, the next procedure FIND_EDGES3 is obtained.

\section{Procedure FIND_EDGES3;}

\section{begin}

1. $G_{1} \leftarrow G ; \pi \leftarrow L F(G) ; i \leftarrow 1 ; E_{0}^{\prime} \leftarrow \emptyset$;

2. while $\pi \neq \emptyset$ do

\section{begin}

3. $\quad$ if $|\pi| \leq 3$ then

4. Find an edge set $E_{i}^{\prime \prime}$ as $E^{\prime}$ in Proposition 12 (1) or [13:

5. else

$$
\text { begin } / *|\pi| \geq 4 * /
$$

6. $\quad$ Find a matching $\mathcal{M}^{\prime \prime}=\left\{\left(v_{2 p-1}, v_{2 p}\right) \mid 1 \leq p \leq m^{\prime}\right\}$ of $R\left(G_{i}\right)$, where if $|\pi| \leq 2 \sigma+6$ then $m^{\prime} \leftarrow\lfloor\pi / 2\rfloor$, otherwise $m^{\prime} \leftarrow \sigma+3$;

7. $\quad$ if $|\pi| \leq 2 \sigma+6$ then

\section{begin}

Choose $E_{s}^{\prime} \subseteq E_{i}^{\prime}$ with $\left|E_{s}^{\prime}\right|=\sigma+3-m^{\prime}$ appropriately; $\mathcal{M}^{\prime} \leftarrow \mathcal{M}^{\prime \prime} \cup\left\{(v, w) \in E_{R} \mid\left(v^{\prime}, w^{\prime}\right) \in E_{s}^{\prime}, v^{\prime} \in Y(v), w^{\prime} \in Y(w)\right\} ;$ 


$$
\begin{aligned}
& \quad /{ }^{*} \mathcal{M}^{\prime} \text { is a matching on } R(G) \text { in the case.*/ } \\
& \text { end; } \\
& \text { else } \\
& \quad \mathcal{M}^{\prime} \leftarrow \mathcal{M}^{\prime \prime} \text {; }
\end{aligned}
$$

8. Find an augmenting pair $E_{i}^{\prime \prime}$ as $\left\{e_{1}^{\prime}, e_{2}^{\prime}\right\}$ in Proposition 24 by choosing $f_{1} \in \mathcal{M}^{\prime \prime} ; \quad \quad / *$ Note that $\left|\mathcal{M}^{\prime}\right|=\sigma+3 . * /$

9. $\quad$ if $f_{j} \in \mathcal{M}^{\prime}-\mathcal{M}^{\prime \prime}$ for $f_{j}$ of Proposition 24 then

begin $/ *$ In the case with $|\pi| \leq 2 \sigma+6 * /$

$E_{i}^{\prime} \leftarrow E_{i}^{\prime}-\left\{\left(y_{2 j-1}, y_{2 j}\right)\right\}, G_{i} \leftarrow G_{i}-\left\{\left(y_{2 j-1}, y_{2 j}\right)\right\}$, where

end;

$$
y_{2 j-1} \in Y_{2 j-1} \text { and } y_{2 j} \in Y_{2 j} \text {; }
$$

10. $E_{i+1}^{\prime} \leftarrow E_{i}^{\prime} \cup E_{i}^{\prime \prime} ; G_{i+1} \leftarrow G_{i}+E_{i}^{\prime \prime}$;

$\pi \leftarrow \pi-\left\{Y(v) \mid v \in V\left(E_{i}^{\prime \prime}\right)\right\} ; i \leftarrow i+1$;

end;

end;

Proposition 25. Any set final $E_{i}^{\prime}$ obtained at the termination of FIND_EDGES3 is a minimum attachment such that $\lambda\left(G^{\prime}\right)=\sigma+1$, where $G^{\prime}=G+E^{\prime}$.

Theorem 2. If $G$ has at least $2 \sigma+6$ leaves then the algorithm FIND_EDGES3 correctly finds a solution $E^{\prime}$ to $(\sigma+1) E C A(S, S A)$ for any given $G$ with $\lambda(G)=\sigma$ in $O\left(\sigma^{2}|V| \log (|V| / \sigma)+|E|+\left|V_{R}\right|^{2}\right)$ time.

\section{Concluding Remarks}

The paper has proposed

(1) an $O\left(\sigma^{2}|V| \log (|V| / \sigma)+|E|+\left|V_{R}\right|^{2}\right)$ time algorithm for finding an optimum solution if $G$ has at least $2 \sigma+6$ leaves or if $\left|\mathcal{L}_{1}\right| \leq\left|\mathcal{K}_{1}\right|$ and $G$ has less than $2 \sigma+6$ leaves,

(2) an $O\left(\sigma^{2}|V| \log (|V| / \sigma)+|E|\right)$ time one for a $\frac{3}{2}$-approximate solution if $\left|\mathcal{L}_{1}\right|>$ $\left|\mathcal{K}_{1}\right|$ and $G$ has less than $2 \sigma+6$ leaves.

We can improve the first algorithm to an $O\left(\sigma^{2}|V| \log (|V| / \sigma)+|E|\right)$ time one by devising how to check whether or not $\left\{f_{1}, f_{2}\right\}$ is an augmenting pair, and whether or not $\mathcal{A}\left(f_{1}, G+\left\{f_{1}, f_{2}\right\}\right)$ is a leaf in Proposition 9 .

\section{Acknowledgments}

The research of T.Watanabe is partly supported by the Grant in Aid for Scientific Research on Priority Areas of the Ministry of Education, Science, Sports and Culture of Japan, under Grant No.10205219. 


\section{References}

1. A. V. Aho, J. E. Hopcroft, And J. D. Ullman, The Design and Analysis of Computer Algorithms, Addison-Wesley, Reading, MA, 1974.

2. J. BANG-JENSEN AND T. JoRDÁN, Edge-connectivity augmentation preserving simplicity, SIAM J. Discrete Math., 11 (1998), pp. 603-623.

3. K. P. Eswaran And R. E. Tarjan, Augmentation problems, SIAM J. Comput., 5 (1976), pp. 653-655.

4. S. Even, Graph Algorithms, Pitman, London, 1979.

5. A. Frank, Augmenting graphs to meet edge connectivity requirements, SIAM J. Discrete Mathematics, 5 (1992), pp. 25-53.

6. H. Frank AND W. Chou, Connectivity considerations in the design of survivable networks, IEEE Trans. Circuit Theory, CT-17 (1970), pp. 486-490.

7. H. N. GaBow, Applications of a poset representation to edge connectivity and graph rigidity, in Proc. 32nd IEEE Symposium on Foundations of Computer Science, 1991, pp. 812-821.

8. — Efficient splitting off algorithms for graphs, in Proc. 26th ACM Symposium on Theory of Computing, 1994, pp. 696-705.

9. T. C. Hu, Integer Programming and Network Flows, Addison-Wesley, Reading, Mass, 1969.

10. T. JORDÁN, Two NP-complete augmentation problems, Tech. Rep. PP-1997-08, Odense University, Denmark, March 1997. http://www.imada.ou.dk/Research/Preprints/j-l.html.

11. A. V. Karzanov And E. A. Timofeev, Efficient algorithm for finding all minimal edge cuts of a nonoriented graph, Cybernetics, (1986), pp. 156-162. Translated from Kibernetika, 2 (1986), 8-12.

12. H. NAGAmochi AND T. IBARAKI, A faster edge splitting algorithm in multigraphs and its application to the edge-connectivity augmentation problem, Tech. Rep. 94017, Kyoto University, 1994.

13. D. Naor, D. Gusfield, And C. Martel, A fast algorithm for optimally increasing the edge connectivity, SIAM J. Comput., 26 (1997), pp. 1139-1165.

14. D. Takafuji, S. TaOka, And T. Watanabe, Simplicity-preserving augmentation to 4-edge-connect a graph, IPSJ SIG Notes, AL-33-5 (1993), pp. 33-40.

15. S. TaOka, D. Takafuji, and T. Watanabe, Simplicity-preserving augmentation of the edge-connectivity of a graph, Tech. Rep. of IEICE of Japan, COMP93-73 (1994), pp. 49-56.

16. S. TAOKA AND T. WATANABE, Efficient algorithms for the edge-connectivity augmentation problem of graphs without increasing edge-multiplicity, IPSJ SIG Notes, AL-42-1 (1994), pp. 1-8.

17. - Minimum augmentation to $k$-edge-connect specified vertices of a graph, in Lecture Notes in Computer Science 834(D-Z du and X-S Zhang(Eds.): Algorithms and Computation), Springer-Verlag, Berlin, 1994, pp. 217-225. (Proc. 5th International Symposium on Algorithms and Computation(ISAAC'94)).

18. _ Smallest augmentation to k-edge-connect all specified vertices in a graph, IPSJ SIG Notes, AL-38-3 (1994), pp. 17-24.

19. R. E. TARJAn, Data Structures and Network Algorithms, CBMS-NSF Regional Conference Series in Applied Mathematics, SIAM, Philadelphia, PA, 1983. 
20. T. Watanabe, An efficient way for edge-connectivity augmentation, Tec. Rep. ACT-76-UILU-ENG-87-2221, Coordinated Science Lab., University of Illinois at Urbana, Urbana, IL 61801, April 1987. Also presented at Eighteenth Southeastern International Conference on Combinatorics, Graph Theory, Computing, No.15, Boca Raton, FL, U.S.A., February 1987.

21. — A simple improvement on edge-connectivity augmentation, Tech. Rep., IEICE of Japan, CAS87-203 (1987), pp. 43-48.

22. T. Watanabe And A. NAKAmura, Edge-connectivity augmentation problems, J. Comput. System Sci., 35 (1987), pp. 96-144.

23. T. Watanabe And M. Yamakado, A linear time algorithm for smallest augmentation to 3-edge-connect a graph, IEICE Trans. Fundamentals of Japan, E76-A (1993), pp. 518-531. 